ESAIM: PROCEEDINGS AND SURVEYS, September 2014, Vol. 45, p. 379-389

J.-S. Dhersin, Editor

\title{
HYPERBOLIC APPROXIMATION OF THE FOURIER TRANSFORMED VLASOV EQUATION
}

\author{
Nhung Pham ${ }^{1}$, Philippe Helluy ${ }^{2}$ and Laurent Navoret ${ }^{3}$
}

\begin{abstract}
We construct an hyperbolic approximation of the Fourier transformed Vlasov equation in which the dependency on the spectral-velocity variable is removed. The model is constructed from the Vlasov equation after a Fourier transformation in the velocity variable as in [9]. A well-chosen finite element semi-discretization in the spectral variable leads to an hyperbolic system. The resulting model has interesting conservation and stability properties. It can be numerically solved by standard schemes for hyperbolic systems. We present numerical results for one-dimensional classical test cases in plasma physics: the Landau damping and the two-stream instability.

Résumé. Nous construisons une approximation hyperbolique de l'équation de Vlasov-Fourier dans laquelle la dépendance dans la variable spectrale associée à la vitesse a été supprimée. Nous commençons par appliquer la transformée de Fourier en vitesse à l'équation de Vlasov comme dans [9]. Puis nous semi-discrétisons l'équation par des éléments finis dans la variable spectrale. Le modèle ainsi construit possède des propriétés intéressantes de conservativité et de stabilité, et peut-être approché par des schémas classiques de résolution des systèmes hyperboliques. Nous présentons des résultats numériques sur des cas tests monodimensionnels classiques en physique des plasmas : l'amortissement Landau et l'instabilité double faisceau.
\end{abstract}

\section{INTRODUCTION}

Solving the Vlasov-Poisson equation is challenging. Some popular methods for studying this equation are the Particle-In-Cell (PIC) method [1] or the semi-lagrangian approach [5].

In a previous work [8], we constructed a reduced Vlasov-Poisson model with a velocity basis expansion.

In this paper, we consider a Fourier velocity transformation of the Vlasov equation. We construct a reduced model where the unknown depends on space and time instead of the full phase-space variables. The reduced model is a linear hyperbolic system, with a non-linear source term. We present numerical results for classical plasma physics test cases.

\section{Plasma mathematical model}

In our work, we consider the one-dimensional Vlasov equation

$$
\partial_{t} f+v \partial_{x} f+E \partial_{v} f=0
$$

${ }^{1}$ IRMA, 7 rue Descartes, 67000 Strasbourg, pham@math.unistra.fr

2 Inria TONUS, philippe.helluy@inria.fr

${ }^{3}$ Inria TONUS, laurent.navoret@math.unistra.fr 
where the unknown distribution function $f$ depends on the space variable $x \in \mathbb{R} / L \mathbb{Z}$, the velocity variable $v \in \mathbb{R}$ and the time variable $t \in \mathbb{R}^{+}$. The electric field $E$ depends on $x$ and $t$ and is the solution of the Poisson equation

$$
\partial_{x} E=-1+\int_{v} f, \quad \int_{x=0}^{L} E=0 .
$$

Equations (1)-(2) are supplemented by an initial condition

$$
f(x, v, 0)=f_{0}(x, v) .
$$

Generally, this initial condition is close to a stationary solution satisfying

$$
\int_{v} f=1
$$

For practical reasons, we will allow that $f$ and $E$ take complex values, however, of course, only the real parts are physically relevant.

We consider a Fourier transformation with respect to the velocity variable (we denote by $I=\sqrt{-1}$ )

$$
\phi(x, \eta, t)=\int_{v=-\infty}^{+\infty} f(x, v, t) \exp (-I \eta v) .
$$

The Fourier velocity variable is denoted by $\eta \in \mathbb{R}$. The distribution function $\phi(x, \eta, t)$ satisfies the Fourier transformed Vlasov equation [9]

$$
\partial_{t} \phi+I \partial_{x} \partial_{\eta} \phi+I E \eta \phi=0 .
$$

In addition, the Poisson equation becomes

$$
\partial_{x} E(x, t)=-1+\phi(x, 0, t) .
$$

We call the new model the Vlasov-Fourier equation. Several conserved quantities are associated to the VlasovPoisson system (see [9]). In particular, the total charge $\rho$, the total energy $\mathcal{E}$ and the $L^{2}$ norm of the distribution function are constant in time

$$
\begin{aligned}
& \rho(t)=\int f(x, v, t) d x d v=\int \phi(x, 0, t) d x, \\
& \mathcal{E}(t)=\frac{1}{2} \int v^{2} f(x, v, t) d x d v+\frac{1}{2} \int E(x, t)^{2} d x=-\frac{1}{2} \int \partial_{\eta^{2}} \phi(x, 0, t) d x+\frac{1}{2} \int E(x, t)^{2} d x, \\
& \|\phi\|_{2}^{2}(t)=\int f^{2}(x, v, t) d x d v=\int \phi^{2}(x, \eta, t) d x d \eta .
\end{aligned}
$$

The Vlasov-Fourier system (3)-(4) enables to numerically better control the high frequencies in velocity [9]. We here present a numerical method for solving this system based on a hyperbolic approximation of the Vlasov equation.

\section{Discretization of the Vlasov-Fourier equation with Respect to the FOURIER VELOCITY VARIABLE}

We will perform a semi-discretization of (3) with respect to the variable $\eta$ in order to obtain a first order hyperbolic system set only in $(x, t)$. We shall call this new system of equations the reduced Vlasov-Fourier model. We could expand the function $\phi$ on a basis of arbitrary functions depending on $\eta$. See for instance [2] and included references. For practical numerical reasons, we decide to choose a classical Lagrange finite element interpolation basis, because it leads to a sparse matrix representation of the hyperbolic system. 


\subsection{Continuous interpolation by the finite element method}

In practice, $\phi$ almost vanishes at the boundaries $\eta \rightarrow \pm \infty$. We consider thus a truncated domain $\eta \in$ $\left[-\eta_{\max }, \eta_{\max }\right]$ and the following dissipative boundary conditions at $\pm \eta_{\max }$

$$
I \partial_{x} \phi\left(x, \pm \eta_{\max }, t\right) \mp \gamma \phi\left(x, \pm \eta_{\max }, t\right)=0,
$$

in which $\gamma \geq 0$. Other boundary conditions could be considered [9].

We recall now how the finite element basis is constructed. We consider an arbitrary polynomial degree $d$. The reference element is defined by

We define the $d+1$ reference nodes by

$$
\hat{Q}=[-1,1]
$$

$$
\hat{N}_{i}=-1+2 \frac{i-1}{d}, \quad i=1 \cdots d+1 .
$$

We mesh the interval $\left[-\eta_{\max }, \eta_{\max }\right]$ with $N$ finite elements $\left(Q_{i}\right)_{i=1 \cdots N}$ and nodes $\left(N_{j}\right)_{j=1 \cdots P}$. The total number of nodes in this interval is $P=d \cdot N+1$. In practice, we suppose that the nodes are equally spaced in $\left[-\eta_{\max }, \eta_{\max }\right]$

$$
N_{j}=-\eta_{\max }+\frac{2 \eta_{\max }}{d N}(j-1)
$$

We introduce a connectivity array for detecting that node $N_{j}$ is the $k^{t h}$ local node of a given element $Q_{i}$

$$
j=\operatorname{connec}(k, i)=k+(i-1) d, \quad 1 \leq k \leq d+1, \quad 1 \leq i \leq N .
$$

We also use the notation

$$
N_{j}=N_{k, i}
$$

and then, element $Q_{i}$ has its support in the interval $\left[N_{1, i}, N_{d+1, i}\right]$.

We construct a transformation $\tau_{i}$ that maps element $\hat{Q}$ onto $Q_{i}$. For this purpose we consider the Lagrange polynomials on $\hat{Q}$, defined by

$$
\hat{L}_{k}(\hat{\eta})=\prod_{l \neq k} \frac{\hat{\eta}-\hat{N}_{l}}{\hat{N}_{k}-\hat{N}_{l}} .
$$

The transformation is then given by

$$
\tau_{i}(\hat{\eta})=\sum_{k=1}^{d+1} \hat{L}_{k}(\hat{\eta}) N_{k, i} .
$$

Because the nodes of the mesh are equally spaced in our application, the transformation $\tau_{i}$ is linear. We construct the interpolation basis in such a way that each basis function $\varphi_{j}$ is associated to a node $N_{j}$ of the mesh and satisfies

$$
\varphi_{j}\left(N_{i}\right)=\delta_{i j}
$$

where $\delta_{i j}$ denotes the Kronecker symbol. We recall how to compute the basis function $\varphi_{j}$. Let $\eta \in\left[-\eta_{\max }, \eta_{\max }\right]$. Necessarily, $\eta$ belongs at least to one finite element $Q_{i}$. Two cases are possible:

(1) Node $N_{j}$ belongs to finite element $Q_{i}$, i.e. $\exists k, N_{j}=N_{k, i}$, then

$$
\varphi_{j}(\eta)=\hat{L}_{k}(\hat{\eta}), \quad \text { where } \eta=\tau_{i}(\hat{\eta})
$$

(2) Node $N_{j}$ does not belong to $Q_{i}$, then

$$
\varphi_{j}(\eta)=0
$$




\subsection{Application to Vlasov-Fourier discretization}

We suppose that the function $\phi(x, \eta, t)$ is well approximated by an expansion on the basis $\left\{\varphi_{j}\right\}_{j=1 \cdots P}$

$$
\phi(x, \eta, t)=\sum_{j=1}^{P} w_{j}(x, t) \varphi_{j}(\eta),
$$

Because of the interpolation property of the basis $\left\{\varphi_{j}\right\}_{j=1 \cdots P}$

$$
\varphi_{i}\left(N_{j}\right)=\delta_{i j}
$$

we have

$$
\phi\left(x, N_{i}, t\right)=\sum_{j=1}^{P} w_{j}(x, t) \varphi_{j}\left(N_{i}\right)=w_{i}(x, t) .
$$

Therefore, we can approximate the initial condition in the following way

$$
w_{j}(x, 0)=\phi\left(x, N_{j}, 0\right)=\phi_{0}\left(x, N_{j}\right) .
$$

Considering equation (3) and boundary condition (6), we can consider the following weak formula of the problem: find $\phi(x, \eta, t)$ such that for all (continuous) test function $\varphi(\eta)$ we have

$$
\begin{aligned}
\int_{\eta} \partial_{t} \phi \varphi+\int_{\eta} I \partial_{x} \partial_{\eta} \phi \varphi+\int_{\eta} I E \eta \phi \varphi & -\frac{1}{2} \varphi\left(\eta_{\max }\right) I \partial_{x} \phi\left(\cdot, \eta_{\max }, \cdot\right)+\frac{1}{2} \varphi\left(-\eta_{\max }\right) I \partial_{x} \phi\left(\cdot,-\eta_{\max }, \cdot\right) \\
& +\frac{1}{2} \varphi\left(\eta_{\max }\right) \gamma \phi\left(\cdot, \eta_{\max }, \cdot\right)+\frac{1}{2} \varphi\left(-\eta_{\max }\right) \gamma \phi\left(\cdot,-\eta_{\max }, \cdot\right)=0 .
\end{aligned}
$$

This "semi-weak" formula is equivalent to initial problem (3) supplemented with boundary conditions (6). Indeed, if $\phi$ is a solution of (3) with conditions (6), it is evident that $\phi$ is also a solution of (11). Reciprocally, if we suppose that $\phi$ is a solution of (11), because (11) is true for arbitrary test function, for every function $\varphi$ such that $\varphi\left(-\eta_{\max }\right)=\varphi\left(\eta_{\max }\right)=0$, we obtain

$$
\int_{\eta} \partial_{t} \phi \varphi+\int_{\eta} I \partial_{x} \partial_{\eta} \phi \varphi+\int_{\eta} I E \eta \phi \varphi=0
$$

and thus (3). Then taking test functions $\varphi$ that do not vanish at $\eta=\eta_{\max }$ or $\eta=-\eta_{\max }$, we obtain the boundary conditions (6).

The factor $1 / 2$ in front of the boundary terms enables to ensure the hyperbolicity of the resulting equation. Indeed, we introduce the following matrices $M, A$ and $B=B_{E}+D$ of dimension $P \times P$, whose coefficients are

$$
\begin{aligned}
& M_{i j}=\int_{\eta} \varphi_{i} \varphi_{j} \\
& A_{i j}=I\left[\int_{\eta} \varphi_{i} \varphi_{j}^{\prime}-\frac{1}{2} \varphi_{i}\left(\eta_{\max }\right) \varphi_{j}\left(\eta_{\max }\right)+\frac{1}{2} \varphi_{i}\left(-\eta_{\max }\right) \varphi_{j}\left(-\eta_{\max }\right)\right] \\
& \left(B_{E}\right)_{i j}=I E \int_{\eta} \eta \varphi_{i} \varphi_{j}, \quad D_{i j}=\frac{1}{2} \gamma\left[\varphi_{i}\left(\eta_{\max }\right) \varphi_{j}\left(\eta_{\max }\right)+\varphi_{i}\left(-\eta_{\max }\right) \varphi_{j}\left(-\eta_{\max }\right)\right] .
\end{aligned}
$$

and we obtain the following equation

$$
M \partial_{t} W+A \partial_{x} W+B W=0
$$


in which $W(x, t)$ is the vector of $P$ components

$$
W=\left(w_{1}, w_{2}, \ldots, w_{P}\right)^{T}
$$

Obviously, the mass matrix $M$ is positive hermitian. An integration by parts in $\eta$ shows that $A$ is hermitian (thanks to the factor $1 / 2$ ). Finally, $B_{E}$ is skew-hermitian and $D$ is diagonal non-negative. It is then classical to prove that system (13) is hyperbolic (i.e. that $M^{-1} A$ is diagonalizable with real eigenvalues [6]) and energy dissipative. Indeed, the $L^{2}$ norm of the distribution function is dissipated

$$
\begin{aligned}
\frac{d}{d t}\|\phi\|_{2}^{2} & =-\int_{x} W(x, t)^{T} D W(x, t) d x \\
& =-\frac{\gamma}{2} \sum_{i=1}^{P}\left[\varphi_{i}^{2}\left(\eta_{\max }\right)+\varphi_{i}^{2}\left(-\eta_{\max }\right)\right] \int_{x} w_{i}^{2}(x, t) d x
\end{aligned}
$$

where

$$
\|\phi\|_{2}^{2}=\int_{x} W(x, t)^{T} M W(x, t) d x=\int_{x, \eta \in\left[-\eta_{\max }, \eta_{\max }\right]} \phi^{2}(x, \eta, t) d x d \eta .
$$

In practice, to compute the matrices $M, A, B$ we use the Gauss-Legendre integration and sparse matrix representations.

\section{Finite volume schemes}

We describe now the numerical approximation. We assume that the spatial domain $] 0, L\left[\right.$ is split into $N_{x}$ cells. The cell $C_{i}$ is the interval $] x_{i-1 / 2}, x_{i+1 / 2}\left[, i=1 \cdots N_{x}\right.$. For practical reasons, we also consider two virtual cells $C_{0}$ and $C_{N_{x}+1}$ for applying the periodic boundary condition. At the beginning of a time step, we copy the values of the cell $C_{N_{x}}$ to the cell $C_{0}$, and the values of the cell $C_{1}$ to the cell $C_{N_{x}+1}$. The space step is $\triangle x=L / N_{x}$ and the center of the cell $C_{i}$ is $x_{i}=(i-1 / 2) \triangle x$. We also consider a sequence of times $t_{n}, n \in \mathbb{N}$, such that $t_{0}=0$ and $t_{n}=n \triangle t$, where $\triangle t$ satisfies the following CFL condition

$$
\triangle t=\alpha \frac{\triangle x \triangle \eta}{2 d \pi}, 0<\alpha \leq 1
$$

This condition is heuristically obtained from the classical CFL condition $v_{\max } \Delta t \leq \triangle x$, and the Shannon sampling condition $\Delta \eta / d=2 \pi / v_{\max }(\triangle \eta / d$ is the sampling interval in the $\eta$ direction).

We consider a finite volume approximation of (13). We denote by $W_{i}(t)$ a piecewise constant approximation of $W$ in each cell

$$
W_{i}(t) \simeq W(x, t), \quad x \in C_{i} .
$$

We obtain the following semi-discrete (in space) approximation

$$
M \partial_{t} W_{i}=-\frac{F\left(W_{i}, W_{i+1}\right)-F\left(W_{i-1}, W_{i}\right)}{\triangle x}-B W_{i} .
$$

where $\left(W_{L}, W_{R}\right) \mapsto F\left(W_{L}, W_{R}\right)$ denotes the numerical flux.

We then introduce a time discretization to compute $W_{i}^{n}$

$$
W_{i}^{n} \simeq W\left(x, t^{n}\right), \quad x \in C_{i}
$$


We use a time second order scheme given by the following algorithm

$$
\begin{aligned}
M \frac{W_{i}^{n+1 / 2}-W_{i}^{n}}{\triangle t / 2} & =-\frac{F\left(W_{i}^{n}, W_{i+1}^{n}\right)-F\left(W_{i-1}^{n}, W_{i}^{n}\right)}{\triangle x}-B W_{i}^{n}, \\
M \frac{W_{i}^{n+1}-W_{i}^{n}}{\triangle t} & =-\frac{F\left(W_{i}^{n+1 / 2}, W_{i+1}^{n+1 / 2}\right)-F\left(W_{i-1}^{n+1 / 2}, W_{i}^{n+1 / 2}\right)}{\triangle x}-B W_{i}^{n+1 / 2} .
\end{aligned}
$$

We consider several choices for the numerical flux $F\left(W_{L}, W_{R}\right)$. We consider the centered flux or a numerical flux with small numerical viscosity ("slightly upwinded flux"). The centered flux is given by

$$
F\left(W_{L}, W_{R}\right)=A \frac{W_{L}+W_{R}}{2},
$$

and the slightly upwinded flux

$$
F\left(W_{L}, W_{R}\right)=A \frac{W_{L}+W_{R}}{2}-\frac{\delta}{2}\left(W_{R}-W_{L}\right)
$$

where $\delta>0$ is the numerical viscosity coefficient.

In practice, for saving CPU time and memory, we use two subroutines for computations with the sparse matrices in skyline format. The first one computes the product of a sparse matrix and a vector. The other one is used to solve a linear system (by the $L U$ method).

\section{TESt CASES}

In this section, we will apply our numerical scheme, (15-16) and (12), on two test cases: the Landau damping and the two stream instability. The electric field, solution of the Poisson equation (4), is computed with the FFT (Fast Fourier Transform) algorithm.

We will compute the charge $\rho$, the total energy $\mathcal{E}$ and the $L^{2}$ norm of the distribution function in the computational domain $[0, L] \times\left[-\eta_{\max }, \eta_{\max }\right]$ see equation (5)). Only the last quantity is exactly conserved (if $\gamma=0$, (see equation (14)). We will also compute the $L^{2}$ norm of the electric field

$$
\|E\|_{2}(t)=\sqrt{\int_{x} E(x, t)^{2} d x} .
$$

We are also interested in the distribution function in physical variable $(x, v)$. The inverse Fourier transform reads

$$
f(x, v, t)=\frac{1}{2 \pi} \int_{-\infty}^{+\infty} \phi(x, \eta, t) \exp (I \eta v) d \eta \simeq \frac{1}{2 \pi} \int_{-\eta_{\max }}^{\eta_{\max }} \phi(x, \eta, t) \exp (I \eta v) d \eta .
$$

We apply the rectangle method with oversampling for computing (17), in order to avoid Shannon aliasing. For this computation we can use a naive DFT computation instead of the FFT algorithm, because this step is applied only at the beginning and the end of the simulation.

In our numerical experiments, the discretization parameters are $N=40, d=5, N_{x}=256$, and $\eta_{\max }=20$.

\subsection{The Landau damping}

In this test case, the initial distribution function and the initial electric field are given by

$$
\begin{aligned}
f_{0}(x, v) & =(1+\varepsilon \cos (k x)) \frac{1}{\sqrt{2 \pi}} e^{-\frac{v^{2}}{2}}, \\
E_{0}(x) & =\frac{\varepsilon}{k} \sin (k x),
\end{aligned}
$$




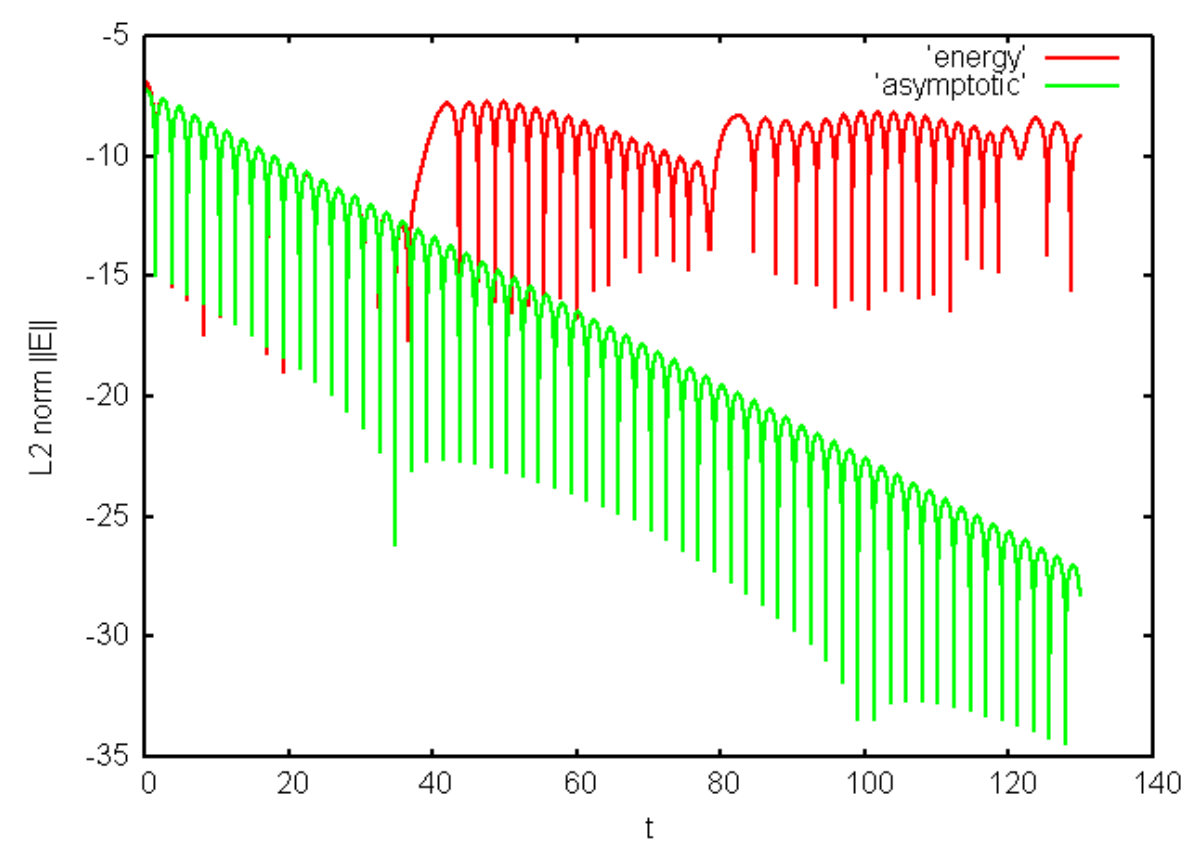

Figure 1. Landau damping test case : $L^{2}$ norm of the electric field $\|E\|_{2}$ as a function of time. The green curve "asymptotic" is the analytical solution and the red curve "energy" is computed with the Vlasov-Fourier method with the slightly upwinded flux $(\delta=0.01, \gamma=5)$.

where $\varepsilon>0$ and $k \in \mathbb{N}^{*}$, and the domain size is $L=2 \pi / k$. So, the initial distribution function in the velocity Fourier variable writes

$$
\phi_{0}(x, \eta)=\int_{-\infty}^{+\infty} f_{0}(x, v) e^{-I \eta v} d v=(1+\varepsilon \cos (k x)) e^{-\frac{\eta^{2}}{2}} .
$$

For small $\varepsilon$, thanks to a linear approximation of the non-linear Vlasov-Poisson system, it is possible to compute an approximate analytical solution of the electric field. The details of the computation are given in [7].

Let us consider the parameters $k=0.5$ and $\varepsilon=2 \times 10^{-4}$. In Figure 1, we compare the time evolution of the $L^{2}$-norm of the electric field obtained by our numerical scheme with the analytical solution. The scheme parameters are chosen as follows: $\delta=0.01$ (slightly upwinded flux) and $\gamma=5$ (dissipative $\eta$ boundary condition). Up to time $t \approx 40=\eta_{\max } / k$, the two curves coincide and then the recurrence phenomena occurs (when the solution reaches the boundary in the $\eta$ direction, cf. [9]). In Figure 2, we observe that the electron charge, the total energy and the squared $L^{2}$ norm are approximately conserved up to the recurrence time.

\subsection{Two-stream instability}

In this test case, the initial distribution function reads

$$
f_{0}(x, v)=(1+\varepsilon \cos (k x)) \frac{1}{2 \sqrt{2 \pi}}\left(e^{-\frac{\left(v-v_{0}\right)^{2}}{2}}+e^{-\frac{\left(v+v_{0}\right)^{2}}{2}}\right),
$$

in which the velocity $v_{0}>0$ is given. It leads to the following initial function $\phi_{0}$ in the Vlasov-Fourier case

$$
\phi_{0}(x, \eta)=(1+\varepsilon \cos (k x)) e^{-\frac{1}{2} \eta^{2}} \cos \left(\eta v_{0}\right) .
$$

The value of parameters for this test case are $k=0.2, \varepsilon=5 \times 10^{-3}$ and $v_{0}=3$. 


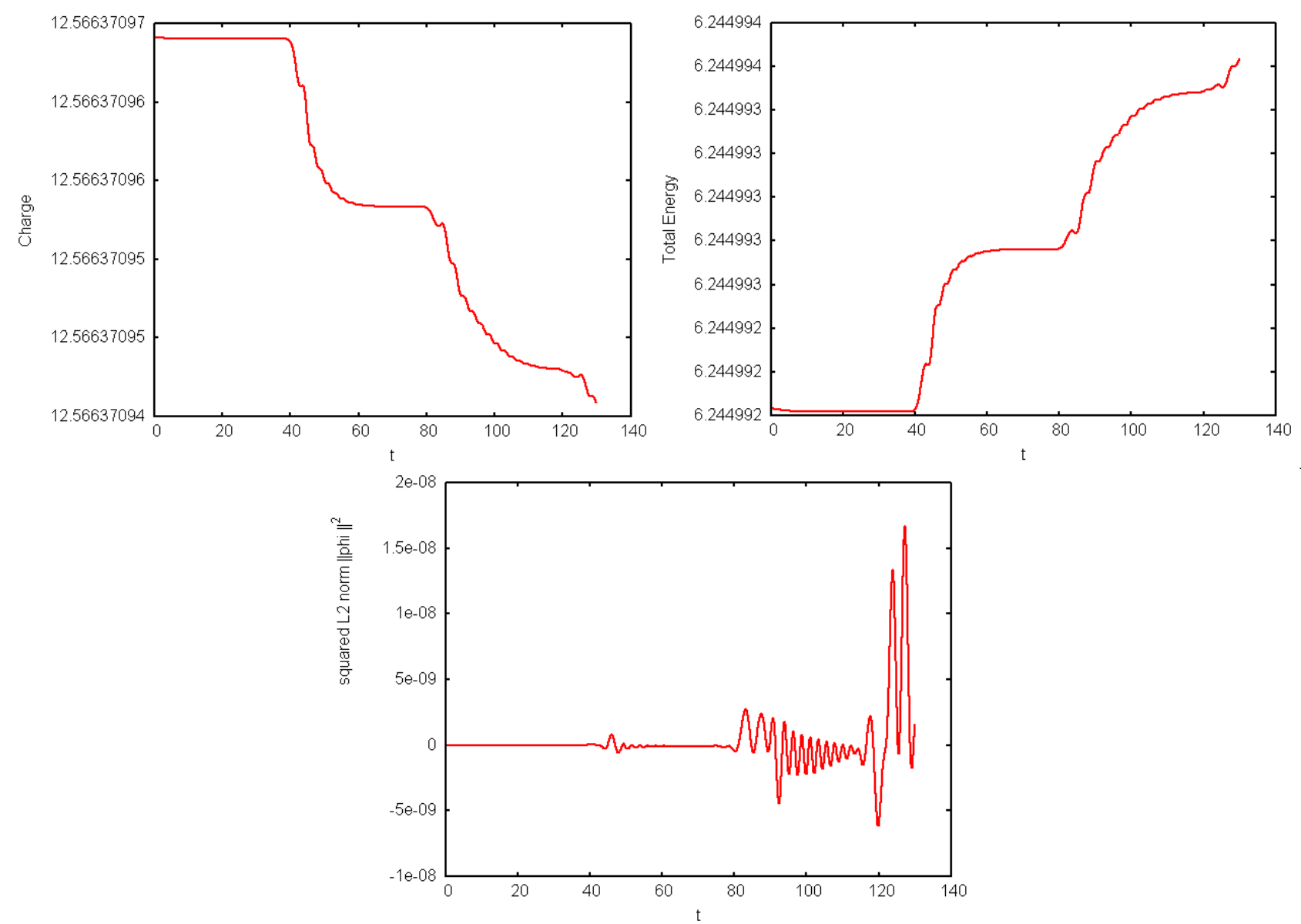

Figure 2. Landau damping test case : charge $\rho$ (top left), total energy $\mathcal{E}$ (top right) and squared $L^{2}$ norm $\|\phi\|_{2}^{2}$ (bottom) as functions of time - computed with the reduced VlasovFourier method with the slightly upwinded flux $(\delta=0.01, \gamma=5)$.

The distribution function is plotted at times $t=25$ and $t=50$ in Figures 3 and 4 . We compare the VlasovFourier method with the slightly upwinded flux $(\delta=0.005$ and $\gamma=0)$ with the Particle-In-Cell (PIC) method. At time $t=50$ (Figure 4), the two methods capture the filamentation of the solution but they are slightly different. Increasing the numerical dissipation when taking $\delta=0.05$ (Figure 4 bottom left), the oscillations are smoothed in the whole domain. Using the boundary dissipation in the $\eta$ direction when taking $\gamma=5$ (Figure 4 bottom right), the oscillations on the edges of the distribution support are removed: they indeed resulted from the $L^{2}$ norm conservation.

The Vlasov-Fourier method with the centered flux is actually unstable. In Figure 5, the real part of the distribution function is displayed. As time increases, oscillations propagate from the $\eta=0$ line to the boundaries $\eta= \pm \eta_{\max }$. For the centered flux, unphysical oscillations develop. In Figure 6, we can observe that the scheme remains unstable even when using the dissipative boundary conditions in the $\eta$ direction.

\section{REFERENCES}

[1] C. K. Birdsall, A. B. Langdon. Plasma Physics via Computer Simulation. Institute of Physics (IOP), Series in Plasma Physics, 1991. 


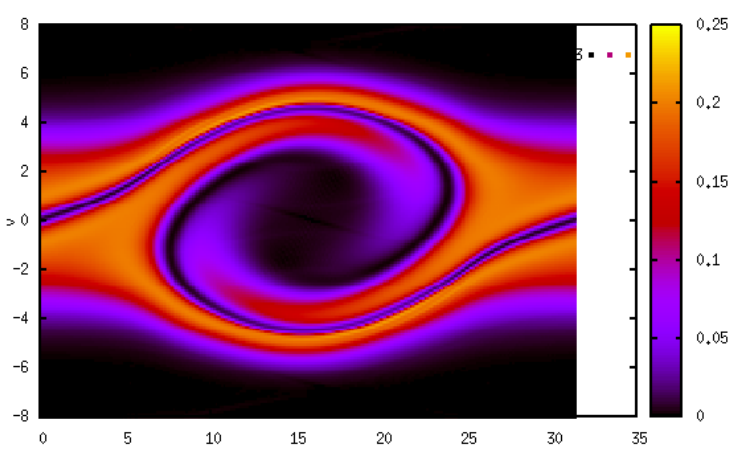

Vlasov-Fourier method $(\delta=0.005, \gamma=0)$

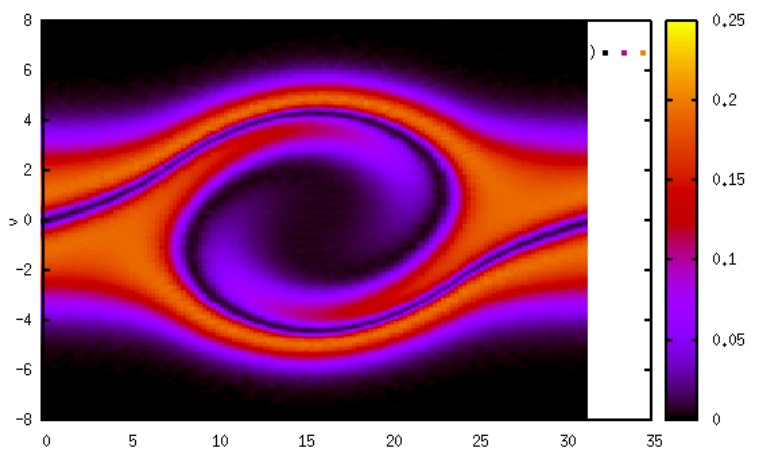

PIC method

FIgURE 3. Two-stream instability test case : distribution function $f(x, v, t)$ at time $t=25$ in the $(x, v)$ phase space. Left: Vlasov-Fourier method with the slightly upwinded flux $(\delta=0.005)$. Right: the PIC method.
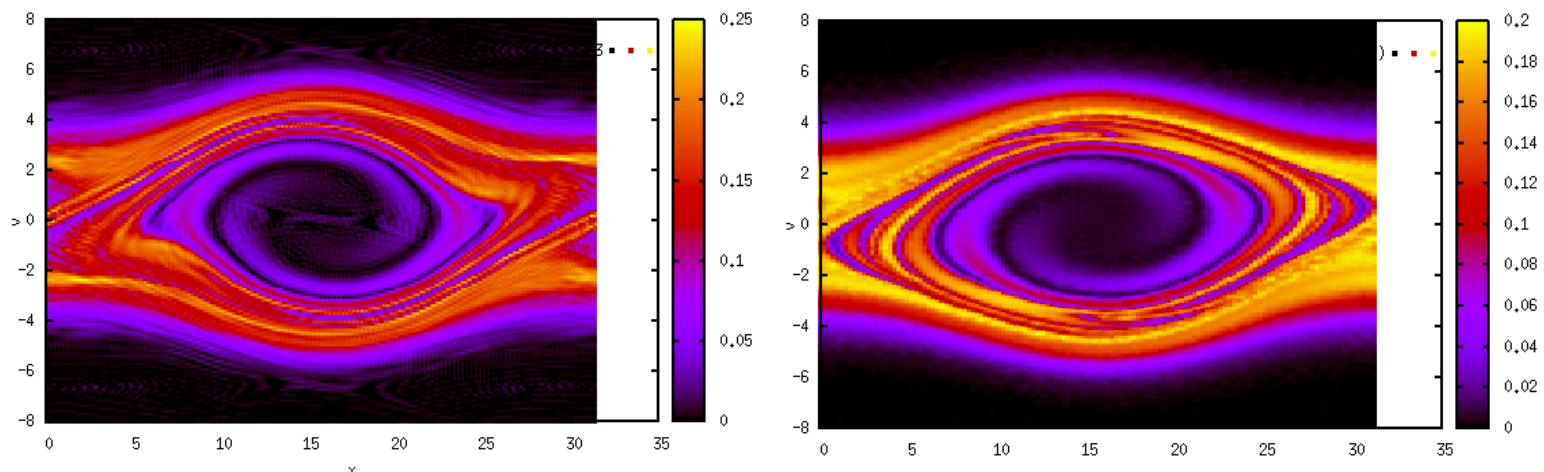

Vlasov-Fourier method $(\delta=0.005, \gamma=0)$
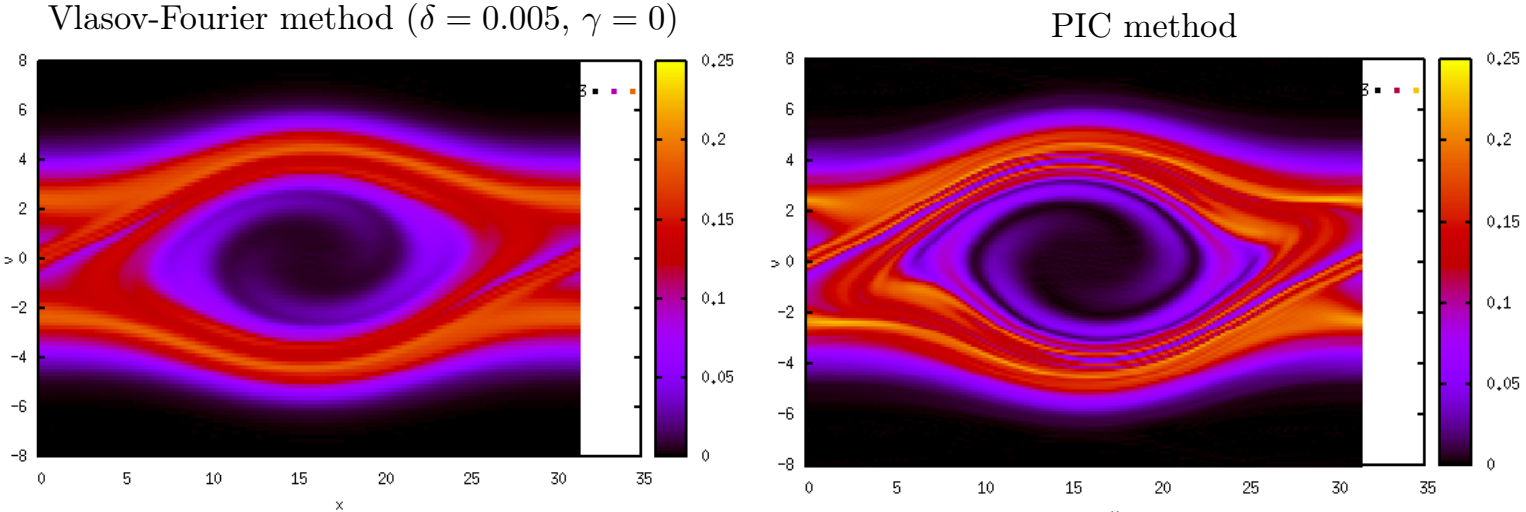

Vlasov-Fourier method $(\delta=0.05, \gamma=0)$

Vlasov-Fourier method $(\delta=0.005, \gamma=5)$

Figure 4. Two-stream instability test case : distribution function $f(x, v, t)$ at time $t=50$ in the $(x, v)$ phase space. Comparison of the Vlasov-Fourier method with the slightly upwinded flux with the PIC method. 


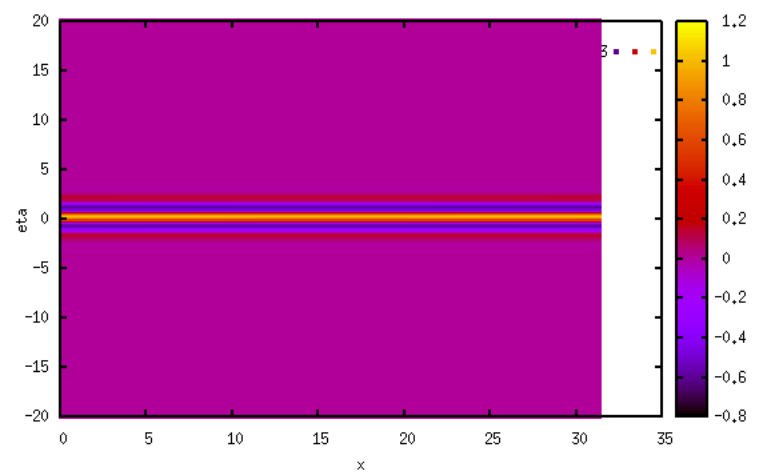

slightly upwinded flux : $t=0$

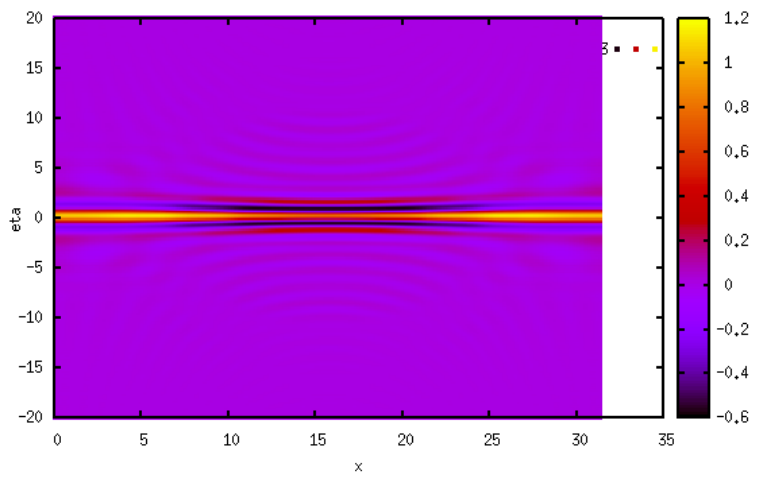

slightly upwinded flux : $t=50$

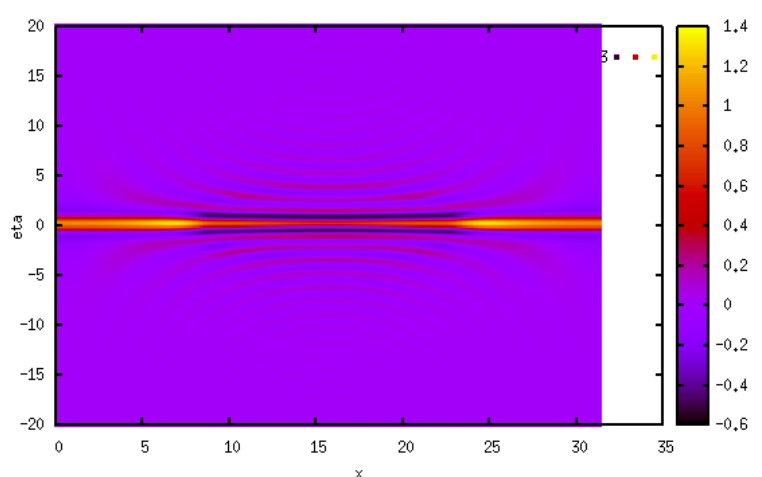

slightly upwinded flux : $t=25$

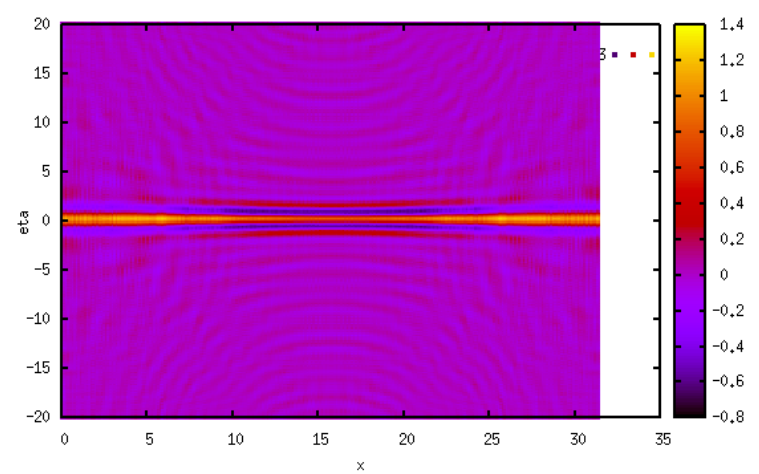

centered flux : $t=50$

FiguRE 5. Two-stream instability test case : real part of the Fourier-transformed distribution function $\Re(\phi(x, \eta, t))$ in the $(x, \eta)$ phase space. Comparison of the slightly upwinded flux $(\delta=0.05)$ with the centered flux for the reduced Vlasov-Fourier method.

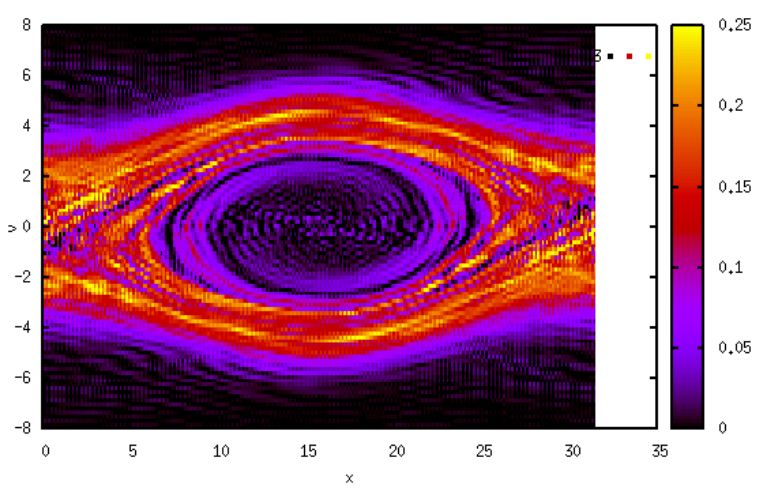

centered flux $(\delta=0, \gamma=0)$

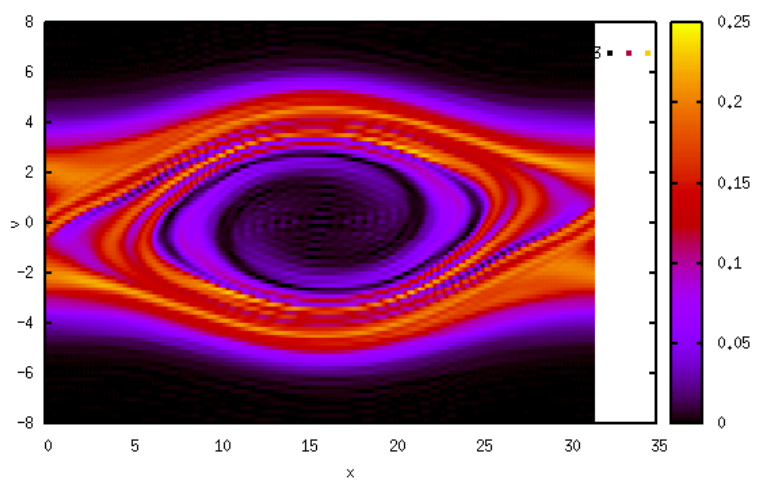

centered flux $(\delta=0, \gamma=5)$

Figure 6. Two-stream instability test case : distribution function $f(x, v, t)$ at time $t=50$ in the $(x, v)$ phase space. The Vlasov-Fourier method using the centered flux with and without dissipative boundary conditions. 
[2] S. Le Bourdiec, F. de Vuyst, L. Jacquet. Numerical solution of the Vlasov-Poisson system using generalized Hermite functions. Comput. Phys. Comm. 175 (2006), no. 8, 528-544.

[3] A. Crestetto. Optimisation de méthodes numériques pour la physique des plasmas. Application aux faisceaux de particules chargées, October 2012.

[4] N. Crouseilles, M. Mehrenberger, E. Sonnendrücker. Conservative semi-Lagrangian schemes for Vlasov equations. J. Comput. Phys. 229 (2010), no. 6, 1927-1953.

[5] F. Filbet, E. Sonnendrücker. Comparison of Eulerian Vlasov solvers Comput. Phys. Commun., 150 (2003), pp. $247-266$.

[6] E. Godlewski, P.-A. Raviart. Numerical approximation of hyperbolic systems of conservation laws. Applied Mathematical Sciences, 118. Springer-Verlag, New York, 1996.

[7] E. Sonnendrücker. Approximation numerique des equations de Vlasov-Maxwell, Notes du cours de M2, 18 mars 2010.

[8] N. Pham, P. Helluy and A. Crestetto. Space-only hyperbolic approximation of the Vlasov equation. CEMRACS 2012, ESAIM: PROCEEDINGS, December 2013, Vol. 43, p. 17-36.

[9] Eliasson, Outflow boundary conditions for the Fourier transformed one-dimensional Vlasov-Poisson system, J. Sci. Comput. , 2001. 